\title{
An Automatic High-Precision Audiofrequency Capacitance Bridge
}

\author{
ROBERT D. CUTKOSKY, FELLOW, IEEE
}

\begin{abstract}
A compact transformer-ratio-arm bridge has been built in which the balance point is automatically determined with the aid of an internal microprocessor. The instrument described in this paper can operate from $20 \mathrm{~Hz}$ to $20 \mathrm{kHz}$, and has three ranges; 12, 120, and 1200 pF. The instrument can resolve one part in $10^{8}$ of full scale above 400 $\mathrm{Hz}$, and can be fully controlled over its IEEE-488 bus interface.
\end{abstract}

\section{INTRODUCTION}

A NUMBER of applications exist for instruments capable of measuring capacitances with very high precision. Included among these are direct applications such as the measurement of dielectric constants and the calibration of precision capacitance standards, but a variety of indirect applications, including the measurement of displacement, temperature, and pressure, are also of great importance.

This paper describes a new audiofrequency $(20 \mathrm{~Hz}$ to $20 \mathrm{kHz}$ ) capacitance bridge which was designed for use in the above areas, and which provides a resolution of one part in $10^{8}$ of full scale in the central and upper parts of its frequency range. The bridge uses such conventional passive-bridge circuitry as relay-switched inductive voltage dividers, but is provided with microprocessor control to yield a self-balancing system with the easy operation of a digital multimeter. The inclusion of a microprocessor made it possible to add a number of features to the instrument that would not have been feasible otherwise, and in addition provided the means for incorporating an IEEE488 bus interface for remote control and data logging. The particular microprocessor used was selected because development equipment for it was available in our laboratory. More recent microprocessors or microcontrollers might otherwise have been considered.

\section{System Overview}

The automatic capacitance bridge is comprised of two units, as shown in Fig. 1, which are called the processor unit and the transformer unit. The two-unit construction helps to isolate the high-level signals present in the latter from the low-level detection circuitry of the former, but both units contain mu-metal shields that together are effective enough so that the units can be mounted one above the other with very little electrical interference. Electrical connections between the two units consist of three coaxial

Manuscript received August 10, 1984; revised February 9, 1985.

The author is with the National Bureau of Standards, Gaithersburg, MD 20899.

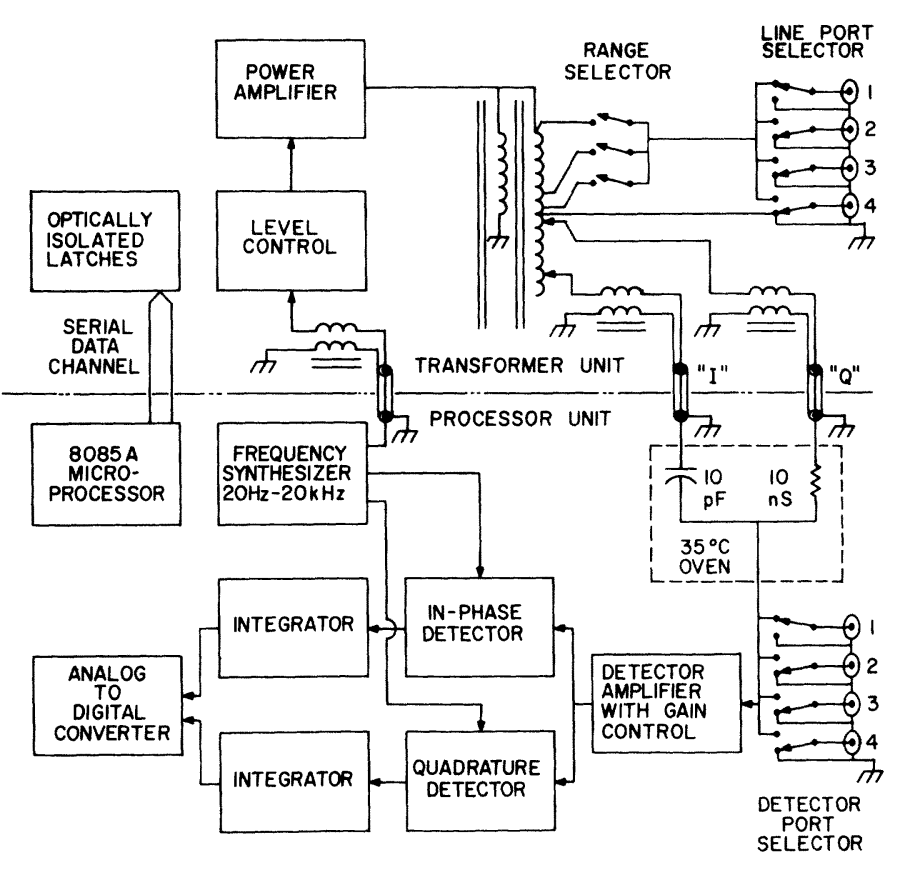

Fig. 1. Schematic diagram of the automatic capacitance bridge.

cables provided with coaxial chokes which are mounted within the transformer unit, and an optically isolated, serial data channel, by means of which the parameters of the transformer unit are controlled.

The microprocessor controls all of the features of the bridge, including the iterative self-balancing procedure, the data formatting, and input-output processing. At the lowest level, the microprocessor provides the timing for all aspects of the bridge operation by means of interrupts, which are obtained from a scaler driven indirectly by the system crystal oscillator, and from a zero-crossing detector connected to the $60-\mathrm{Hz}$ power line. The timing functions are necessary for the proper operation of the frequency synthesizer and the detector integrators, which will be described in the next section.

Port selectors are provided at both the line side and the detector side of the bridge. Each selector independently connects one port, and shorts the remaining three ports to ground, so that up to 16 external three-terminal capacitors can be connected to the instrument and measured independently of each other. The line port selector is connected to one of three taps on the "unknown" side of the bridge transformer through a range selector, giving full scale ranges of about 12,120 , and $1200 \mathrm{pF}$. The detector 
port selector is connected to the detector terminals of the reference capacitance and reference conductance standards, and to the summing point of an operational amplifier having capacitive feedback, which constitutes the detector's first stage. This configuration causes the input to be at ground potential, and makes the gain and phase shift of the system independent of the ground capacitance of the unknown capacitor.

The "reference" side of the bridge transformer is effectively an independent pair of inductive dividers. The capacitance or I channel is a 22-bit binary divider that connects to a $10-\mathrm{pF}$ capacitance standard in the processor unit, and the conductance or $\mathrm{Q}$ channel is a 14-bit binary divider with a 128-times smaller voltage range that connects to a 10-nS conductance standard in the processor unit.

The measurement process that is performed automatically by the bridge can be considered to consist of three steps: 1) selecting and applying a voltage to the transformer, and measuring the detector offset with a suitable gain; 2) calculating, based on internally stored constants, revised settings for the inductive dividers, and displaying the result of the measurement; and 3) iterating. Before each iteration the transformer voltage is increased by a factor not greater than about 32, up to the maximum that can be supported at the operating frequency, but limited so that the voltage on the unknown capacitor does not exceed a maximum that may be entered through the keyboard or over the IEEE-488 bus. The detector gain is then increased, if necessary, to achieve an overall sensitivity increase of about 32, up to the maximum gain allowed at the operating frequency. Five iterations or fewer are required to advance from the lowest to the highest sensitivities, a process that requires up to $15 \mathrm{~s}$. After reaching the highest sensitivity, and provided that the previous relay settings need not be changed, the bridge recycles and provides new measurements at a rate slightly slower than once per second.

The balancing scheme requires that the gain and phase shift of the system, measured from the power amplifier through the unknown capacitor, detector, and integrator, be known and stable to about two percent. A look-up table consisting of a 2048-byte erasable read-only memory (ROM) is contained in the bridge to hold the gain constants. Four constants are maintained for every available gain setting, at each of 29 selected frequencies. The microprocessor interpolates between the frequencies listed within the table to obtain the values that apply at the operating frequency. The table itself is generated during the construction or realignment of the bridge through the use of a program that runs on a desk calculator that is connected to the bridge over the IEEE- 488 bus. For this series of measurements, the bridge is operated in a special calibration mode. The table generation routine requires about $5 \mathrm{~h}$ of virtually unattended operation. Most of this time is spent in obtaining many measurements at each data point, so that highly reliable average values can be used in the calculations.

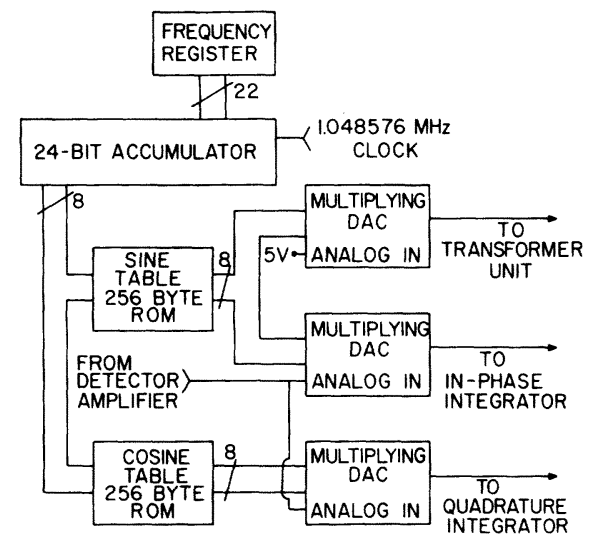

Fig. 2. Block diagram of the frequency synthesizer and digital phase-sensitive detector.

\section{Frequency Synthesis, Phase-Sensitive Detection, AND Timing}

The frequency synthesizer contained in the bridge is a conventional all-digital design [1] using a clocked accumulator and a sine look-up table, as shown in the upper part of Fig. 2. Each time a clock pulse occurs, the number that was stored in the frequency register by the microprocessor when the bridge operating frequency was selected is added to the number that is in the accumulator. The most significant eight bits of the accumulator select the address of a sine look-up table. The digital output of the sine table is converted to an analog sinewave using a digital-to-analog converter (DAC) consisting of a multiplying DAC in which the analog input is held to $+5 \mathrm{~V}$. Not shown in the figure are the deglitching and filter circuits associated with the converter.

The in-phase phase-sensitive detector was constructed, as indicated in Fig. 2, using a second muiltiplying DAC in which the analog input is the ac output of the detector. The output of this DAC is equal to the analog signal multiplied by the digital sine function, which when integrated over an integral number of periods produces a value that is dependent only upon the fundamental frequency sinusoidal component of the analog signal, plus a dc offset. The inclusion of a cosine table and a third multiplying DAC followed by another integrator provides an output proportional to the fundamental frequency cosinusoidal component of the analog signal.

In order for the above scheme to operate correctly, the integrators should operate for an integral number of periods, and the dc offsets of the components beginning at the output of the detector amplifier and including the offset of the analog-to-digital converter (ADC) following the integrators, should cause no errors. Integration over an integral number of cycles is provided by detecting the beginning of each sine wave, which occurs when the highorder address bit of the sine table goes from high to low. These transitions are counted in a scaler that is preset by the microprocessor at the same time that the frequency register is set. The preset count is calculated so that the scaler recycles in about $0.1 \mathrm{~s}$. Each time the scaler recy- 
cles, the microprocessor is interrupted. The microprocessor starts and stops the integrators within the interrupt service routine, thus assuring that the integrations are over an integral number of periods. Each integration occupies four scaler cycles, or about $0.4 \mathrm{~s}$. The integrator outputs are normalized by the processor to the nominal 0.4-s period by multiplying the digital output by a correction factor, but this is only significant at very low frequencies.

The dc offsets present in the phase-sensitive detectors are canceled by performing a second integration, identical with the first; except that during the second integration, the phase of the signal is reversed within the transformer unit. Simply subtracting the digital representations of the integrator outputs eliminates all dc offset errors, and results in a total effective integration time of $0.8 \mathrm{~s}$.

The principle interfering signals in a low-frequency bridge are the fundamental and harmonics of the power line, which is at $60 \mathrm{~Hz}$ in this laboratory. Some problems have also been observed here at power line subharmonics as low as $15 \mathrm{~Hz}$, but only when measuring very low voltage signals. In order to eliminate these interfering signals, the start of the sinewave generation after each phase reversal is synchronized with a $15-\mathrm{Hz}$ pulse train derived from a zero-crossing detector connected to the $60-\mathrm{Hz}$ power line. This is accomplished by clearing and disabling the accumulator in the frequency synthesizer after each integration, and reenabling it after the occurrence of the next $15-\mathrm{Hz}$ pulse. The system is allowed to run until the next scaler interrupt is detected before the integrators are started, which gives a settling time of about $0.1 \mathrm{~s}$. A complete iteration in the measurement cycle, therefore, takes place in about $1.0 \mathrm{~s}$, plus the time spent waiting for two $15 \mathrm{~Hz}$ interrupts; assuming that the inductive divider relays are not changed. If the microprocessor program calls for a revised inductive divider relay setting, an additional delay is provided to allow the switching transients to settle.

The phase-sensitive detection scheme outlined above is sufficiently free from harmonic sensitivities that it was not necessary to tune the detector to improve its ability to reject harmonics. So long as the bridge is used only in applications that are not strongly frequency dependent, which includes capacitance measurements and inductive divider calibrations, but which may not include resonance methods for measuring inductances, no problems are to be expected.

\section{Transformers}

The stability, linearity, and general utility of audiofrequency bridges based on magnetically shielded threewinding transformers have been recognized for many years [2], [3]. A three-winding transformer with good magnetic shielding between the excitation winding and the ratio winding, and with tight coupling between the sections of the ratio winding, has no significant magnetic errors. The errors in such a transformer are caused by capacitive currents between the ratio windings, and from the windings

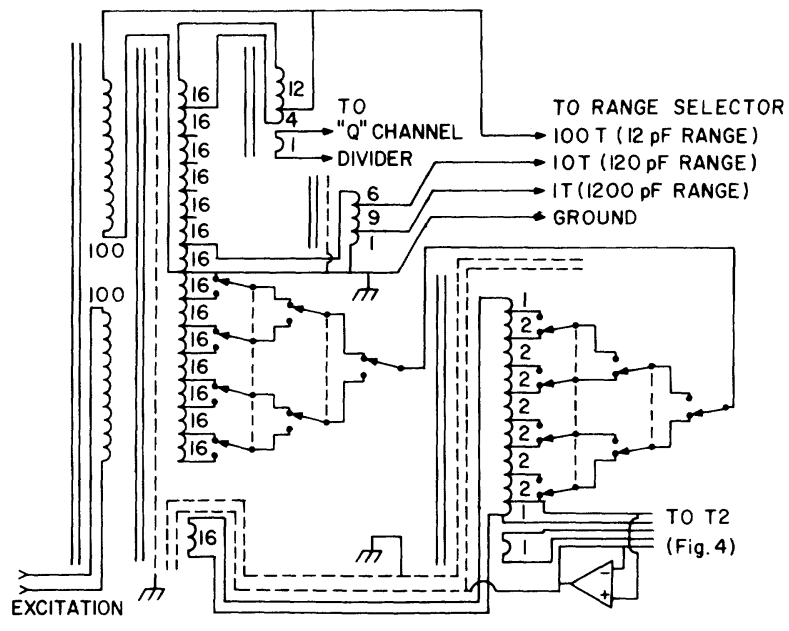

Fig. 3. Wiring of the main bridge transformer $T 1$. (Each winding encircles all cores directly to its left, and no others. Numbers by windings indicate number of turns.)

to ground. Roughly speaking, the capacitive errors are proportional to the capacitive admittances multiplied by the winding impedances, both of which are reduced by keeping the number of turns on the ratio windings to a minimum. Reducing the number of turns reduces the sensitivity of a bridge using such a transformer, unless the transformer has a very large and expensive core. Electrostatic shielding between the ratio windings may be used to control the interwinding capacitances, but this technique must be used with great caution, because it can increase the winding impedances so much that the external loads on the transformer become a serious problem, and it can reduce the coupling between the ratio windings to such a degree that the magnetic errors of the transformer are no longer negligible.

All of the above factors enter into the design of the transformer system for any transformer-ratio-arm bridge. An automatic bridge has an additional aspect that must be considered; namely, that the resistances of the switches cannot easily be made as low as is possible with a manual bridge. The bridge described here uses relays with resistances between 0.015 and $0.030 \Omega$, dependent upon how many contacts are in parallel; which is nearly an order of magnitude larger than can be obtained from a manual switch. One good return from the use of relays is the freedom to mount them without considering the appearance of the front panel.

The essential features of $T 1$, the main bridge transformer, are shown in Fig. 3. This is a multistage transformer, in which the first stage consists of two bifilar windings of 100 turns each, occupying a single layer around the first core. The second-stage winding consists of a bundle of wires wrapped 16 times around the first stage, the second core, and a toroidal mu-metal shield. One wire of the bundle is doubly shielded, and drives one of the three independent third-stage windings to form the lower three of the most significant 6 bits of the 22-bit I-channel divider. Two other independent third-stage windings are employed as shown to adapt the binary di- 
vision scheme of the reference side of the divider to the decimal scheme needed for the range selector. Strapping the second 100-turn first-stage winding to the composite 100 -turn ratio winding as it enters the $12-\mathrm{pF}$ range selector effectively eliminates the impedance in series with this terminal of the three-winding transformer equivalent circuit of $T 1$, but at the expense of doubling the impedance in series with the reference side of the transformer. The circuit was wired as indicated because the load presented to the reference side is constant and controllable, whereas the load presented to the unknown side is the unknown capacitance itself with its ground and cable capacitance. When the $120-\mathrm{pF}$ range is selected, the unknown-side series impedance is given by a complex expression that depends upon the turns ratio. The effect is that the unknownside series impedance of $T 1$ is smaller than the winding impedance by about a factor of ten. When the 1200-pF range is selected, the unknown-side series impedance is almost entirely attributable to the resistance of the single turn on $T 1$ and the interconnecting wires.

The third-stage winding associated with the I-channel divider is enclosed in a double electrostatic shield. The outer shield is grounded, and the inner is connected to the output of a voltage follower, as shown in Fig. 3. These connections are extended to the doubly shielded secondstage winding that drives this third-stage bundle. With the input to the voltage follower connected to the third-stage output as indicated, all displacement currents within the transformer that change with changes in the relay positions, assuming that the capacitive loads to ground in the following stages have been eliminated, are restricted to the region between the two concentric shields. If the driving point connections to the shields are properly placed, this shielding system greatly reduces the errors due to magnetic couplings into the ratio windings from displacement currents that depend upon the divider tap selected, and produces an adjustable-ratio transformer in which the total error is a simple sum of the errors associated with the individual windings.

The errors due to the loading effects of the second-stage interwinding capacitances were canceled by connecting selected capacitors between the taps of the second-stage winding and an auxiliary transformer (not shown) excited from one of the first-stage windings. In effect, the currents in the interwinding capacitances are supplied through the auxiliary transformer and the selected capacitors, rather than through the ratio winding itself. Only the most significant three bits of the I-channel voltage divider, and no part of the Q-channel divider, required compensation.

The lower 16 bits of the I-channel divider are provided by a pair of 8-bit inductive dividers in cascade. The first of these 8-bit dividers $T 2$, is a three-stage design with magnetic shielding between the first and second stages, but the second $T 3$, has only two stages, with no magnetic shield. These dividers are contained within an electrostatic shield that is driven from the voltage follower shown in Fig. 3.

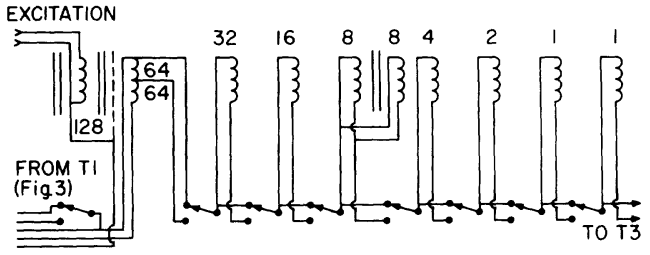

Fig. 4. Wiring of the first cascaded transformer $T 2$. (The notation is the same as in Fig. 3.)

A schematic drawing of the three-stage divider $T 2$, is shown in Fig. 4. Note that the most significant bit is obtained from a one-turn winding on $T 1$, so that in reality only 7 bits are provided by $T 2$. The most significant three of these bits are provided by a set of windings constructed from a bundle of wires that encircles the first- and secondstage cores eight times. The least significant four bits of the transformer are obtained from a winding formed from a single-turn bundle over the third core, and strapped to the second stage. The first stage of $T 2$ is excited by an auxiliary transformer driven from one of the first-stage windings of $T 1$.

The output of $T 2$ is connected to $T 3$, which is similar to $T 2$, except that it has no separately excited first stage or magnetic shield. Unfortunately, only seven bits could be obtained by copying the wiring of $T 2$, so that another factor of two is required. Doubling the number of turns on all windings would have provided a solution, but at the cost of increasing the series impedances of the transformer by a factor of four. The approach taken was to use the same number of turns that was used on $T 2$, but to add a "half-turn" winding for the last bit, by using two cores of half the height that would otherwise have been used, in place of a single core; and by passing this last winding around one core only. Strapping the two cores together with one or more low-impedance links in the form of a figure " 8 " forces the flux in the two cores to be equal, and also eliminates the excessive leakage inductance that would otherwise be associated with the fractional turn. This technique can be extended in principle to produce any rational ratio, and was applied here to a transformer that has in reality two stages, if the core linking the singleturn bundle is considered.

The output of the I-channel divider is connected to a coaxial choke followed by a coaxial cable leading to the $10-\mathrm{pF}$ reference capacitor in the processor unit, and is also connected to a current pump [4], which is an operational amplifier configured to exhibit a negative capacitance between a terminal and ground. The synthesized negative capacitance is adjusted to eliminate the total load at the output of the divider.

The 14-bit Q-channel divider is constructed as a pair of 7-bit dividers. They are similar to the 8-bit dividers used in the I-channel divider, but are not provided with a driven shield. The Q-channel divider (second stage) is excited from a single turn on one of the stage-three windings of the main transformer, as noted in Fig. 3. The Q-channel divider does not use a current pump. 


\section{Reference Standards}

The $10-\mathrm{pF}$ reference capacitor was constructed by grinding a disk of Suprasil II grade fused silica to the dimensions shown in Fig. 5; and firing gold electrodes on the surfaces, and in the slot around the edge. The slot electrode is connected to ground, and the electrodes on the two surfaces form the active electrodes of the capacitor. Fused-silica capacitors can be exceptionally stable, and are far more rugged than air capacitors [5]. The major difficulty encountered in their construction is the elimination of voltage dependencies, which in exceptional cases can cause the capacitances to change by several parts in $10^{7}$ when the voltage changes from a few volts to 100 or $200 \mathrm{~V}$. The construction technique that was employed is still under development, but the capacitor used in this bridge, although a very early model, changes less than one part in $10^{9}$ for voltages below $200 \mathrm{~V}$, and is now drifting at the rate of a few parts in $10^{7}$ per year. The techniques employed will be described in a forthcoming paper.

The 10-ns conductance standard consists of a $100-\mathrm{M} \Omega$ film resistor in a glass envelope that was mounted between two parallel guard plates with holes in their centers through which the resistor was passed. The spacing between the guard plates matches the length of the resistive film on the resistor, which is located so that the resistive film is in the region between the plates. One of the plates is connected to one end of the resistor, and the other plate is grounded. This configuration produces a conductance standard with a tolerably small phase angle at frequencies below $20 \mathrm{kHz}$. Although a 10 -ns conductance connected directly to the detector contributes more Johnson noise than is desirable, the alternative of using a 1-nS conductance standard would have increased the phase angle of the standard to an unacceptable level. Some other alternatives were rejected because of their complexity.

Fused-silica capacitors have temperature coefficients around $10 \mathrm{ppm}$ per degree Celsius. It is necessary to control the temperature of these capacitors to about $0.001^{\circ} \mathrm{C}$ if the uncertainties in capacitance are to be smaller than a few parts in $10^{8}$. The temperature-controlled oven of the bridge maintains the reference capacitor at a temperature near $35^{\circ} \mathrm{C}$, constant to about $0.001^{\circ} \mathrm{C}$ per month. A fourterminal resistance thermometer that is separate from the control sensor is contained in the regulated oven to provide a means for monitoring the internal temperature. The thermometer leads are brought out to a jack on the front panel of the bridge.

A cross section of the regulated oven is shown in Fig. 6 . The upper section containing the conductance standard is an appendage to an oven that was designed originally to house a capacitance standard only. The temperature control within it is adequate, but not outstanding. The section of the oven containing the capacitance standard was designed so that the lag between changes in the heater power and the response at the control sensor was minimized, which is necessary so that a high-gain amplifier can be used in the feedback loop to provide close temper-

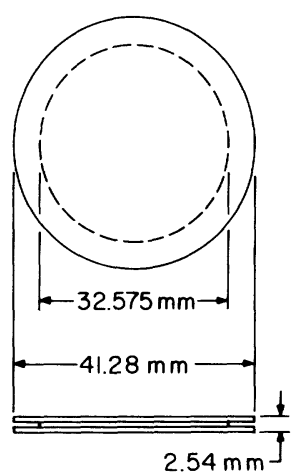

Fig. 5. Dimensions of the fused-silica element used to make the $10-\mathrm{pF}$ reference capacitor.

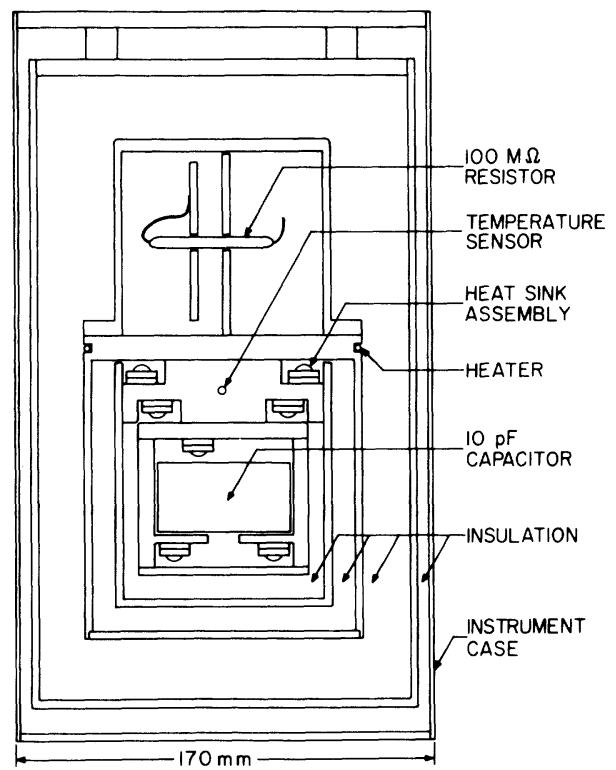

Fig. 6. Assembly drawing of the constant temperature oven for the capacitance and conductance standards. (The structure is mostly of aluminum, with foam insulation. Shielded leads are brought into the enclosure from the top, through thin-wall stainless steel tubes.)

ature control. The oven was also designed so that temperature gradients in its exterior walls would be strongly attenuated before reaching the capacitance standard, and so that the temperature difference between the capacitance standard and the control sensor would be negligible.

The control circuit used with the regulated oven has been described elsewhere [6], and has been in use here for several years. It makes use of a $100-\Omega$ platinum resistance thermometer for the control sensor, and a four-arm bridge. The other three arms are hermetically sealed resistors located within the regulated oven. The bridge is driven with $15-\mathrm{Hz}$ square-waves, and the out-of-balance signal is amplified with an ac amplifier before it is synchronously demodulated, integrated, and routed to the heater. The temperature-regulator bridge is provided with the equivalent of double Wagner arms to suppress the effects of conductances from the leads to ground. 


\section{Calibration and Use}

Normally, the output display of the capacitance bridge is a pair of decimally coded numbers that represent effective values for the two sets of binary dividers. These effective values are obtained by the microprocessor by adding each actual divider setting to the offset obtained from the in-phase and quadrature phase-sensitive detectors through calculations involving the gain and phase shift of the detector. The gain and phase-shift figures are stored in a look-up table in ROM, as was described in Section II. The table values are relied upon to increase the measurement precision from that yielded directly by the 22-bit divider, to that of the smallest displayed digit, which is one part in $10^{8}$ of full scale. The microprocessor converts the effective values for the binary dividers from binary to decimal for display, but uses the binary form to select the inductive divider relay settings for the next measurement iteration. The relays are not updated if the bridge sensitivity is at its maximum, and if the indicated changes for both dividers are either no more than one step, or are smaller than about four times the expected standard deviation of a measurement. This increases the life of the relays by preventing them from changing needlessly during repeated measurements, and increases the rate of repeated measurements. The expected standard deviations are stored with the gain table in the ROM.

Sometimes it is useful to know which inductive divider taps were involved in a measurement, so that the results of a measurement series can be examined for systematic errors, so that corrections can be applied to the divider settings, if necessary, and so that unambiguous external calibrations can be performed to assess the bridge linearity. If the appropriate command is sent to the bridge over the IEEE- 488 bus, the bridge will append the inductive divider settings, in ASCII-coded hexadecimal format, to all of its usual data transmissions on the bus. The local display on the panel of the bridge is not affected by this feature.

Preliminary measurements have been made of the bridge linearity, which indicated that, with the capacitive compensation technique outlined in Section IV, the bridge will differ from linearity by about one part in $10^{7}$ of full scale, for frequencies below $3500 \mathrm{~Hz}$. These measurements were made in a semimanual mode, in which the bridge was operated through a program on a desk calculator, but an operator was required to be present to connect and disconnect the external capacitance standards used with the measurement technique. An automatic system is now under construction that will provide for connecting one or more capacitors from a group of ten to the bridge, individually or in parallel; with provisions for increasing the load capacitances by appropriate factors to allow extrapolation to no-load conditions. The process used is a modification of an earlier technique that involved permuting a set of capacitors [7]. Combining this system with a capacitive current divider to change its range is expected to

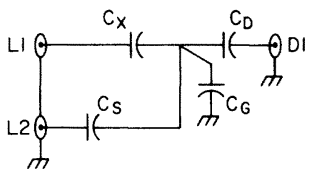

(a)

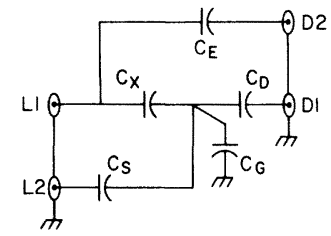

(b)
Fig. 7. Connections used to measure large capacitors with the automatic bridge (see text).

provide a means for automatically calibrating any of the bridge ratios that may be desired.

Although this bridge normally measures only to 1200 $\mathrm{pF}$, with some additional effort it can be used to measure much larger capacitances. It is not possible to extend the range through the use of a transformer, however, because the detector input is the summing point of an operational amplifier, and a high input capacitance causes instability or excessive noise. Generally, it is best to limit the total capacitance to ground at the detector port selector to 2500 $\mathrm{pF}$ or less.

One technique that has been used to extend the range of the bridge is illustrated in Fig. 7(a). The large capacitor to be measured is called $C_{X} ; C_{S}$ is a known, stable threeterminal standard, typically 100 or $1000 \mathrm{pF} ; C_{D}$ is a threeterminal capacitor of $1000 \mathrm{pF}$ or less, with good shortterm stability; and $C_{G}$ represents all of the capacitance to ground at the junction of $C_{X}, D_{D}$, and $C_{S}$. If we let $C_{A B}$ be the capacitance measured from line port $A$ to detector port $B$, and remember that all unselected ports are grounded, we have

$$
C_{11}=C_{X} C_{D} /\left(C_{X}+C_{D}+C_{S}+C_{G}\right)
$$

and

$$
C_{21}=C_{S} C_{D} /\left(C_{X}+C_{D}+C_{S}+C_{G}\right) .
$$

It follows that $C_{X}=C_{S} C_{11} / C_{21}$.

The largest source of uncertainty in this measurement is the unstable and unknown impedance of the short at port $L 1$ when port $L 2$ is selected. In effect, the impedance of the short at port $L 1$ is included in the measurement of $C_{X}$. One way of reducing this uncertainty is through the circuit of Fig. 7(b), which is similar to Fig. 7(a), but with the addition of capacitor $C_{E}$, of $1000 \mathrm{pF}$ or less. The principle used with Fig. 7(b) is to select and hold line port $L 2$, so that the impedance of the short at port $L 1$ is kept constant. One can then measure the separate responses at ports $D 1$ and $D 2$. The latter measurement gives a transfer admittance proportional to the impedance of the short at port $L 1$. The calculated value for $C_{X}$ can then be corrected, which gives very nearly the four-terminal-pair value for $C_{X}$ [8]. An approximate value for $C_{E}$ is required, and can be obtained by selecting ports $L 1$ and $D 2$.

The technique described above has been used to measure $10-\mu \mathrm{F}$ capacitors automatically, at frequencies up to 10 
$\mathrm{kHz}$, giving standard deviations ranging from 10 to 60 $\mathrm{ppm}$. Capacitors as large as $100 \mu \mathrm{F}$ have been measured in this way up to $20 \mathrm{kHz}$, using an external port selector having lower impedances than the internal line ports. Surprisingly, the technique may be least reliable when it is used to measure relatively small capacitors. In such cases, or whenever the ground capacitance $C_{G}$ is a significant part of $\left(C_{X}+C_{D}+C_{S}+C_{G}\right)$, care must be taken to be sure that the voltage dependence of $C_{G}$ does not cause an error.

\section{ConClusion}

This paper has described an automatic capacitance bridge that is expected to find use in a wide variety of areas. Many of the techniques used in the bridge may be useful in other high-precision instruments as well, such as automatic resistance and inductance bridges. It is believed that in the case of this capacitance bridge, the principle advantage gained through automation has been to provide a system that can be fully calibrated with the aid of a desk calculator or minicomputer, routinely and without significant operator intervention.

\section{ACKNOWLEDGMENT}

The author wishes to thank Lai H. Lee of the NBS Electricity Division for his invaluable assistance and support while this work was underway.

\section{REFERENCES}

[1] J. Tierney, C. M. Rader, and B. Gold, "A digital frequency synthesizer," IEEE Trans. Audio Electroacoust., vol. AU-19, pp. 48-56, Mar. 1971.

[2] A. M. Thompson, "The precise measurement of small capacitances," IRE Trans. Instrum., vol. I-7, pp. 245-253, Dec. 1958.

[3] R. D. Cutkosky, "Techniques for comparing four-terminal-pair admittance standards," NBS J. Res., vol. 74C, pp. 63-78, July-Dec. 1970.

[4] A. C. Corney, "A universal four-pair impedance bridge," IEEE Trans. Instrum. Meas., vol. IM-28, pp. 211-215, Sept. 1979.

[5] R. D. Cutkosky and L. H. Lee, "Improved ten-picofarad fused silica dielectric capacitor," NBS J. Res., vol. 69C, pp. 173-179, July-Sept. 1965.

[6] R. D. Cutkosky and R. S. Davis, "Simple control circuit for temperature regulation and other bridge applications," Rev. Sci. Instr., vol. 52, no. 9, pp. 1403-1405, Sept. 1981.

[7] R. D. Cutkosky and J. Q. Shields, "The precision measurement of transformer ratios," IRE Trans. Instrum., vol. I-9, pp. 243-250, Sept. 1960.

[8] J. Q. Shields, "Measurement of four-pair admittances with two-pair bridges," IEEE Trans. Instrum. Meas., vol. IM-23, pp. 345-352, Dec. 1974 .

\title{
A Digital AC Bridge as an Impedance to Frequency Converter
}

\author{
HARTMUT SCHOLLMEYER
}

\begin{abstract}
This paper deals with an automatic digital ac bridge, used as an impedance to frequency converter. The fundamental principle, including the balance equations and the circuitry, is described. Besides measuring impedances the ac bridge can be used as an interface device for passive sensors.
\end{abstract}

\section{INTRODUCTION}

$\mathrm{I}^{\mathrm{N}}$ N MANY applications, variations of impedances, e.g. sensors' impedances, are transduced into frequencydependent output signals which contain the information in the form of frequency variations. Cost-effective digitizing of these signals is feasible without any considerable errors if they are slowly varying which facilitates their integration into superior computer systems.

Manuscript received June 8, 1984; revised August 27, 1984.

The author was with the Institute für Allgemeine Elektrotechnik, Technische Universität Berlin, Berlin, Germany. He is now with the Hochschule der Bundeswehr Hamburg, Fachbereich Elektrotechnik, Hamburg, Germany.
Usually impedances are measured by direct conversion methods, limiting the measurement accuracy built-up of systematic errors. This unconventional ac bridge is a selfbalancing device, which combines the advantages of the bridge method and the direct conversion method. It avoids drift errors as a consequence of its digital organization.

\section{Fundamental Method of the AC Bridge}

This ac bridge transforms the values of the impedances into a proportional frequency-dependent output signal (Fig. 1). The four elements of the circuit are formed by two symmetrical digitally synthesized sine wave generators [2] with equal voltage amplitudes, and by the two impedances to be compared. The bridge is automatically balanced by means of a detector, which acts to shift the frequency of both ac sources $\left(U_{D}=0\right)$. There is no need for switches or final control elements to induce balance, i.e., the bridge remains untouched, and all stray capac- 